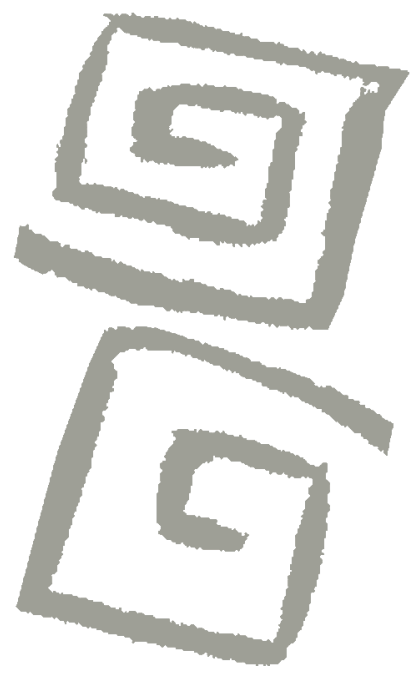

\title{
El sujeto de los riesgos en un mundo transhumano y posclínico: reflexiones a partir de Todos los nombres de Saramago y de Matrix de las hermanas Wachowski
}

\author{
Subjects at risk in a transhuman and post-clinical world: \\ Reflections on Saramago's All the Names and the \\ Wachowski Sisters' The Matrix
}

Naomar Almeida-Filho ${ }^{1}$

${ }^{1}$ Doctor en Epidemiología. Profesor Visitante, Instituto de Estudios Avanzados, Universidade de São Paulo. Profesor Titular Senior Instituto de Saúde Coletiva, Universidade Federal da Bahia. Investigador I-A, Conselho Nacional de Desenvolvimento Científico e Tecnológico $(C N P q) . \triangle$ iD
RESUMEN La premisa principal de este texto es que el discurso social común, expresado en las artes y las humanidades, ha sido crucial para el proceso de construcción de lenguajes y cosmologías tecnocientíficas. Se examinaron los fundamentos y las correlaciones de este argumento tomando como referencia disciplinas científicas establecidas de manera reciente, como la epidemiología, con la ayuda de dos obras de arte: la novela Todos los nombres, escrita por Saramago, y la trilogía cinematográfica Matrix, escrita y dirigida por las hermanas Wachowski. Ambas alegorías son ilustrativas del mundo virtual posibilitado por la tecnociencia. La propuesta consiste, entonces, en establecer un paralelo entre los primeros observatorios sociales que encapsularon poblaciones enteras para su observación sistemática (lo que permitió el perfeccionamiento de la metodología epidemiológica) y el "sueño epidemiológico", casi cumplido con la introducción del procesamiento electrónico de información y potenciado ahora por el avance de estrategias de modelado y simulación y por la organización de megabases de datos de salud, enfermedad, vida y muerte.

PALABRAS CLAVES Teoría Epidemiológica; Riesgo; Literatura; Cine.

\begin{abstract}
The main premise of this paper is that common social discourse, manifested in the Arts \& Humanities, has played a crucial role in the construction of technoscientific languages and cosmologies. I explore this argument in relation to recently established scientific disciplines, such as Epidemiology, through the lens of two works of cultural production: Saramago's novel All the Names, and cult movie trilogy The Matrix, written and directed by the Wachowski Sisters. Both are allegories that exemplify a virtual world made possible by technoscience. A parallel is suggested between the first social observatories that encapsulated whole populations for systematic observation (allowing improvement of epidemiological methodology) and the "epidemiological dream" - nearly realized through the introduction of electronic data processing, enhanced by the advancement of modeling and simulation strategies and the organization of immense databases on health, disease, life, and death.
\end{abstract}

KEY WORDS Epidemiological Theory; Risk; Literature; Films. 


\section{INTRODUCCIÓN}

Mi premisa es que los investigadores de todas las áreas podrían recurrir a las artes y las humanidades para verse mejor a sí mismos y a sus ciencias a través del espejo de la sociedad y la cultura. El discurso social común, expresado en productos culturales y artísticos, constituye sin dudas una valiosa fuente de imágenes, metáforas y sentidos para la construcción de cosmologías de referencia y de lenguajes tecnocientíficos imprescindibles para las ciencias, sobre todo, para las que se constituyen en la formación epistemológica contemporánea que Esther Díaz ${ }^{(1)}$ denomina "posciencia". Sin embargo, en este camino de actualización permanente del lenguaje científico en idiomas disciplinarios y transdisciplinarios no se debe esperar que el imaginario social y el simbólico-cultural estén representados de manera instantánea o sin mediaciones ${ }^{(2)}$.

En el campo de la salud, particularmente en lo que concierne a la epidemiología, el lenguaje del riesgo ha sido instrumental para la creación de un mundo propio, sui generis, peculiarmente demarcado, sobre todo mediante objetos-modelo epidemiológicos determinados por las funciones de riesgo, los factores (y marcadores) de riesgo y, más recientemente, los comportamientos de riesgo ${ }^{(3)}$. En este campo científico, los fundamentos del pensamiento y del lenguaje se muestran incluso más cruciales en la medida en que la centralidad del riesgo, en el imaginario de la sociedad actual, impone cada vez más a los epidemiólogos y sanitaristas una mayor comprensión y mejores respuestas para los enigmas de la salud( ${ }^{(4)}$.

Provocado por el entonces recientemente publicado Manifiesto Cyborg de Donna Haraway ${ }^{(5)}$, escribí en 1991, en Berkeley, un pequeño ensayo titulado "El hombre de los riesgos", el cual fue publicado primero en La clínica y la epidemiología ${ }^{(6)}$, y luego ampliado e integrado a un volumen sobre temas de epistemología de la epidemiología, Ilamado La ciencia tímida ${ }^{(7)}$. En ese momento y contexto, propuse que el concepto epidemiológico de riesgo produce discursos capaces de construir mundos ficticios formados por poblaciones abstractas, y que la noción clínica de riesgo individual puebla esos universos con simulacros de personas, definidos por sus probabilidades de enfermar y de morir, y agregados en grupos humanos conectados por redes sociales virtuales. La hipótesis central era que, en ese mundo digital vaticinado, los "hombres de riesgos" se materializarían en dimensiones paralelas bajo condiciones propias de producción-reproducción de efectos sobre la vida humana y transhumana, mediante procesos socio-históricos que implicarían una existencia virtual y, en términos de salud, una morbilidad cyborg correspondiente. Sevalho ${ }^{(8)}$ y Portella et al ${ }^{(9)}$ revisitaron el texto a partir de diferentes perspectivas críticas e indicaron analogías y contrastes con la idea de "hombre lento", acuñada originalmente por Milton Santos.

A partir de las controversias generadas por diferentes aportes críticos a lo largo de estas casi tres décadas, y considerando las nuevas interfaces de la epidemiología y las transfiguraciones de las formaciones sociales en el capitalismo cognitivo ${ }^{(10)}$, pretendo actualizar en estas páginas la proposición original del "hombre de riesgos" a una noción de "sujeto de riesgos". Teniendo en cuenta el avance de los estudios de género y la superación de los límites impuestos por la modernidad, ya no tiene sentido utilizar el término "hombre" para abarcar a toda la diversidad humana. Me refiero a personas posthumanxs o ya transhumanxs, si bien todavía son modernxs, que se encuentran inmersxs en ecologías de saberes y prácticas de predicción, prevención y precaución de riesgos, enfermedades, sufrimiento y muerte, y de promoción de la salud y de la vida. En este mundo transcartesiano y posclínico, que constituye una distopía ensombrecida por la necropolítica, hay lugar para una perspectiva nueva (o reloaded, como veremos a continuación) de aquel sujeto como "ser transhumano de los riesgos".

Para esto, realizo mi propuesta a partir de dos temas centrales de la epistemología contemporánea aplicados a la ciencia epidemiológica: primero, el lenguaje como elemento fundamental para la construcción de mundos 
científicos; y segundo, el conocimiento racional constituido por esa práctica social Ilamada ciencia, que se encuentra sometido a las determinaciones de un mundo que se supone real y que está cada vez más naturalizado, aunque es ciertamente histórico ${ }^{(11,12)}$. En principio, esta proposición puede sonar extraña y tal vez incomprensible, en particular, para investigadores-artesanos y científicos "duros" acostumbrados a tratar con fenómenos de lo que se conoce comúnmente como vida real, casos concretos, experimentos controlados, muestras poblacionales, toneladas de registros y volúmenes de datos. En este ensayo, profundizaré y ampliaré este argumento con la ayuda de dos productos culturales del mundo occidental contemporáneo: Todos los nombres, novela considerada un clásico de la literatura portuguesa actual, y Matrix (1999-2003), trilogía de películas que se volvió de culto a inicios del corriente siglo XXI.

\section{TODOS LOS NOMBRES DE SARAMAGO}

Todos los nombres es una obra maestra del escritor portugués José Saramago, Premio Nobel de Literatura en 1998. Trata sobre la Conservaduría General del Registro Civil de una ciudad anónima que, aunque está localizada en algún lugar de Portugal, bien podría situarse en España, Brasil, Cuba o cualquier otro país de herencia iberoamericana ${ }^{(13)}$. En 2004, hice un primer análisis de la premisa principal de este texto, tomando como fundamento la obra de Saramago. Ese artículo fue publicado en una revista científica del mainstream epidemiológico $^{(14)}$ y, como no tuvo gran repercusión, lo reitero y lo amplío acá.

La Conservaduría es el arquetipo de las organizaciones burocráticas tradicionales, las cuales tienen funciones casi oscuras que dependen completamente de reglas anticuadas cuyo origen fue olvidado. Unos oficiales rigurosos e inflexibles, sin cara y casi siniestros, gobiernan y ensombrecen la severa y conservadora institución. Como queda claro en los primeros capítulos, la protagonista del libro es la institución que, con su carácter totalitario, cierne sus pesares sobre los seres humanos. El párrafo inicial de Todos los nombres está enteramente dedicado a la entrada de la Conservaduría:

Encima del marco de la puerta hay una chapa metálica larga y estrecha, revestida de esmalte. Sobre un fondo blanco, las letras negras dicen "Conservaduría General del Registro Civil”. El esmalte está agrietado y desportillado en algunos puntos. La puerta es antigua, la última capa de pintura marrón está descascada, las venas de la madera, a la vista, recuerdan una piel estriada. Hay cinco ventanas en la fachada. Apenas se cruza el umbral, se siente el olor del papel viejo. ${ }^{(13)}$

El primer capítulo del libro incluye una descripción detallada de la Conservaduría, su estructura jerárquica, su modo de operar y, sobre todo, su extraña arquitectura. El salón principal es amplio y rectangular, con muebles de madera maciza, austera, elegante y sobria. Ocho escritorios ubicados cerca del mostrador de atención al público son utilizados por los ayudantes de escribiente, cuatro escritorios corresponden a los escribientes jurados que controlan el trabajo de los ayudantes y otros dos a los oficiales que supervisan a los escribientes jurados. En el centro, al fondo del salón, "solo, como tenía que ser", se encuentra el escritorio grande y oscuro del conservador en jefe. Todo esto "acata naturalmente las precedencias jerárquicas, siendo, como se esperaría, armonioso desde este punto de vista, y también desde el punto de vista geométrico, lo que sirve para probar que no existe ninguna contradicción insalvable entre la estética y la autoridad"(13).

Un principio simple rige el trabajo en la Conservaduría: se debe completar el mayor número posible de tareas dentro de cada nivel, pasando a los niveles superiores de la jerarquía solo una proporción mínima. En condiciones normales de funcionamiento, no se debe molestar al jefe de la Conservaduría con tareas rutinarias o problemas cotidianos del trabajo, con el objeto de garantizar el orden, 
la disciplina y la dignidad necesarios para la gran responsabilidad cívica de la institución.

Todos los nombres es también la historia de Don José, un humilde ayudante de escribiente jurado que, en su primera aparición, es presentado al lector bajo la rúbrica de "insignificancia". En una odisea patética y casi heroica, José se mete en grandes apuros al quebrar todas las reglas de la sombría y cruel institución para intentar descubrir quién era la persona detrás de un registro particular. Sin embargo, no es la vida subterránea de José lo que nos interesa como investigadores, epidemiólogos y científicos. Aunque pueden ser valiosos para otros discursos científicos, las narrativas identitarias y los análisis de relaciones de poder en burocracias conservadoras insinuados en esta alegoría no son pertinentes en este momento.

Lo más importante para nuestro propósito es la función del extraño edificio, en particular, la del pabellón gigantesco donde se almacenan los documentos archivados. Justo detrás del escritorio del Conservador comienzan cinco filas largas de grandes estanterías que van del piso al techo, las cuales prosiguen hasta el final del edificio (aunque no se ve debido al tamaño enorme de la construcción y a la oscuridad en la parte de atrás del corredor). "Obedeciendo la ley de la naturaleza", los archivos y registros se almacenan en dos secciones: los vivos y los muertos. Los documentos relativos a los vivos se archivan en las primeras secciones de las filas de estanterías y contienen todos los nombres y todas las firmas correspondientes. Incluyen desde certificados de nacimiento, permisos de trabajo, certificados de bautismo y licencias de matrimonio, hasta matrículas escolares, diplomas y certificaciones, solicitudes de ausencia por accidentes y enfermedad, premios y jubilaciones. Los documentos relacionados con los muertos, certificados de óbito abrochados a sus archivos de cuando estaban vivos, se arrojan a las secciones más distantes de las estanterías.

Como el número de personas muertas no deja nunca de aumentar, la administración de la Conservaduría ordena, en forma periódica, la demolición de la pared de la parte de atrás del pabellón y la reconstruye varios metros más lejos. La insólita construcción cuenta ya con cientos de metros en los que, para mayor seguridad y conservación del contenido, no hay ventanas ni puertas. En el pasado se alcanzó cierto equilibrio entre los archivos de los vivos y los de los muertos, lo que posibilitó absorber el pequeño aumento de población viva por medio de la compresión física y luego usando tapas cada vez más finas en los expedientes. A los recién nacidos se los archiva al comienzo de las hileras de estanterías, y se va empujando a los más viejos hacia adelante y hacia arriba, hasta que llegan al fondo y a la cima de los estantes superiores. Los registros de quienes se mueren en cualquier momento de su ciclo de vida son transferidos de las secciones de los vivos hacia el comienzo de las largas estanterías de los muertos. Por razones obvias, los archivos de los vivos están mucho mejor organizados que los que pertenecen a los difuntos.

Con todos los esfuerzos realizados para expandir el edificio (ya inmenso y lleno de estanterías gigantescas), se dejaron en el piso numerosas pilas de documentos que bloquearon corredores y formaron senderos y desvíos que, en su conjunto, hicieron del área de archivos una especie de laberinto oscuro y siniestro. Antes de la adopción obligatoria del hilo de Ariadna para marcar el recorrido, un científico que hacía investigaciones heráldicas se perdió por una semana "en las laberínticas catacumbas de los archivos de los muertos"(13).

La Conservaduría abarca el microcosmos de toda una ciudad y una región, y encapsula a su población entera, presente y pasada, viva y muerta. Cada habitante de la municipalidad anónima tiene derecho a un expediente en unos archivos que contienen el registro meticuloso de "todos los nombres" (padre, madre, parientes, autoridades, padrino, madrina, socios comerciales, cónyuges, etc.). Esto implica una existencia paralela para cada habitante dentro de la inmensa base conformada por los documentos capturados en el laberinto de estanterías, archivos y nombres, y cultivada por el cuidado y la negligencia de los ayudantes, escribientes y oficiales. Esta existencia 
paralela permite que los expedientes y fichas de seres de la vida real tengan una "vida autónoma" con relativa independencia en el espacio interno de la patética institución.

\section{LA TRILOGÍA DE MATRIX}

La trilogía de Matrix (The Matrix, 1999; The Matrix Reloaded, 2003; The Matrix Revolutions, 2003), escrita y dirigida por las hermanas Wachowski, alcanzó una gran popularidad a principios de este siglo. Las películas de esta serie presentan una narrativa frenética y compleja en la que el telón de fondo es una guerra entre humanos y robots inteligentes en un futuro distópico cuya fecha no conocemos pero que parece incómodamente cercano. Las máquinas vencieron y dominan a los humanos, y los convirtieron en la cosecha principal de granjas o fábricas biológicas. En ellas, se cultivan miles de millones de personas, manteniéndolas vivas dentro de placentas artificiales para generar la energía electroquímica con la que se alimenta una red enorme de computadoras. Para engañar a los humanos esclavizados y mantenerlos físicamente inertes y neurológicamente activos (aunque controlados), las máquinas crearon un mundo real virtual denominado "Matrix".

La Matrix es un mundo de fantasía generado por computadora, capaz de producir una "simulación neurointeractiva" que consiste, en síntesis, en un entorno virtual totalmente inclusivo. En este entorno, unos seres virtuales poderosos y casi indestructibles Ilamados "agentes" previenen el disenso, controlan rebeliones, y persiguen y destruyen cualquier resistencia. La mayoría de los humanos del mundo real tiene conexiones cerebrales implantadas para acceder al mundo onírico inducido neurológicamente. Al conectarse a la Matrix, ellos se convierten en cyborgs (es decir, org-anismos cyb-ernéticos, híbridos de máquina y organismo vivo), lo que les permite existir en ambos mundos. Neo, el héroe, es un hacker talentoso que, guiado por Morfeo y con la ayuda de un grupo de ciberpartisanos, "despierta del sueño de la realidad".
Salvado de las manos de los agentes, Neo renace en una nave del mundo real comandada por Morfeo para asumir el papel del mesías carismático esperado durante mucho tiempo para liderar la rebelión de los humanos.

La población virtual de seres transhumanos comparte el entorno de la Matrix y, excluyendo a los agentes, se muestra completamente representativa de una población de referencia total. Cada ser humano virtual en la Matrix es un personaje o un avatar, simulacro personalizado de un ser humano real que se encuentra inerte en su cápsula. Estos seres humanoides virtuales, como la mujer de vestido rojo de la primera película o varios personajes de la segunda (como el cerrajero o Merovingian, el líder de la pandilla), son simulacros -término proveniente del latín que significa: "algo que tiene solo la forma o apariencia de una cosa sin poseer su sustancia o cualidades; una mera imagen, una imitación engañosa o una semejanza de algo"(15) - sin contraparte en el mundo real, y por eso la interacción humana con ellos se revela limitada.

Matrix significa "matriz", término originario del latín arcaico del que deriva la palabra "madre", cuyo significado original es "hembra embarazada"(16). En el latín medieval, matrix pasó también a significar "útero" $y$, en una connotación totalmente diferente, "lista" o "registro" de todos los nombres de un grupo (el verbo "matricular" surge de la palabra "matrícula", o pequeña matriz). En distintos contextos, la palabra "matriz" designa un tipo de sustrato en el que nacen, se crían, crecen y se desarrollan diferentes seres. Se puede encontrar en las matemáticas un significado técnico de "matriz", entendida como cualquier disposición ordenada de valores y símbolos. En las diferentes películas de la trilogía, la palabra matrix designa tanto al sistema concreto (maquinaria y programas) que contiene al conjunto de humanos que vegetan en sus receptáculos, como a la secuencia de símbolos que codifica el entorno virtual. En la trilogía, la apariencia de las cosas en el mundo supuestamente real, es decir la Matrix, es una percepción inferior; el entorno real (el "desierto de lo real") constituye la verdadera secuencia matriz de símbolos. 
Las películas de la franquicia Matrix se basan en un argumento fundamental: lo que experimentamos como realidad no es más que un entorno virtual interactivo y artificial, que implica algún tipo de camuflaje global sistemático generado por una mega computadora conectada en forma directa con las mentes de todos los habitantes del mundo. Sin embargo, hay una conexión fundamental entre el mundo real y el mundo simulado por computadora: la naturaleza de un cuerpo transhumano mediado por dispositivos tecnológicos, en particular teléfonos vintage o smartphones hipermodernos (para la época).

Finalmente, dado que aun en los mundos virtuales los órganos de los sentidos son receptores de datos y procesadores de información, el problema lógico (y práctico) no reside en que estos datos sean discontinuos, sino que sean indiscernibles. Por alguna oscura razón, los seres humanos reales pueden conservar recuerdos del mundo virtual, tanto dentro de la Matrix como cuando regresan al mundo real. Esto es lo que les concede a los simulacros de seres humanos una trágica pérdida de autonomía en el interior de los mundos programados, que son distopías totalmente digitales.

Dos décadas después del lanzamiento de este relato tecno-pop, no se puede negar su potencial anticipativo, incluyendo una gran cantidad de previsiones confirmadas que van desde el detalle de los smartphones como portales entre las dimensiones del mundo sombrío y árido ("el desierto de lo real") y el imaginario programado de la vida actual hiperurbana, hasta los ambientes de realidad virtual determinados por algoritmos de inteligencia artificial.

La saga fue un gran éxito de taquilla y rápidamente se convirtió en lo que la crítica denomina una película de culto, representativa de subculturas que se identifican con los valores de la incertidumbre, el misticismo, la fragmentación y los modismos tecnocientíficos. En su conjunto, estas películas configuran un relato ambicioso sobre temas centrales de la filosofía occidental con una estética de videojuego de artes marciales, referencias a la contracultura cyberpunk y algunos rasgos de la cultura new age. Por este motivo, aun después de dos décadas, la alegoría de Matrix continúa despertando el interés de varios pensadores críticos del escenario posmoderno o contemporáneo tardío ${ }^{(17)}$.

Inmediatamente después del lanzamiento de la primera película, Slavoj Žižek ${ }^{(18)}$ propuso que la Matrix representaría el orden simbólico virtual de la cultura humana contemporánea, en una referencia no explícita a la noción castoridiana de institución imaginaria de la sociedad ${ }^{(2)}$ Para Manzotti ${ }^{(19)}$, desde un enfoque con una remota inspiración psicoanalítica, se trata de una red mental que estructura la realidad para todos nosotros y que, para obedecer a su función onírica, necesita permanecer inconsciente.

Hanley ${ }^{(20)}$ define la Matrix como un entorno virtual totalitariamente comunitario que es lo suficientemente grande como para ser universal y que incluye todo el inventario de participantes humanos. Bostrom ${ }^{(21)}$ ve en la Matrix una síntesis de formas distópicas de relación social, en interacción virtual permanente, radical y totalizante. Desde esta perspectiva, la Matrix parece real precisamente debido a su interactividad radical, posibilitada por la capacidad de un entorno programado para responder a cualquier aporte o estímulo de sus elementos. Los entornos virtuales al alcance de la tecnología disponible en la actualidad (como los dispositivos individuales o los simuladores microambientales de realidad virtual) están lejos de este grado de interactividad.

\section{LA CAVERNA DE PLATÓN Y EL "SUEÑO EPIDEMIOLÓGICO"}

Tanto la Conservaduría como la Matrix pueden entenderse, sin dudas, como poderosas metáforas políticas. Los modelos reducidos del mundo real, controlados, rigurosamente delimitados, programados y disciplinados, propios de la mega red universal y de la increíble institución conservadora, tienen un carácter distópico y bien pueden representar la summa de control social y psicológico de la burocracia y del totalitarismo. 
Hay que tener en cuenta que Portugal y la generación de Saramago sufrieron mucho con la dictadura salazarista, el régimen fascista más largo de la historia (que comenzó en 1932 y fue derrocado en 1974 por la Revolución de los Claveles). Como complemento de estas correlaciones políticas, la novela también trata sobre la subversión humana, tal como se mencionó antes, representada por Don José, quien desafía desde adentro de la institución a las fuerzas de la opresión institucional. A su vez, a pesar de no contener una referencia política inmediata como el fascismo salazarista, las películas de la saga Matrix también tratan sobre la acción humana clandestina, heroica y desafiante de las fuerzas de la opresión institucional sin rostro (o, en este caso, con un único rostro: el del Agente Smith). En este sentido, Paura $^{(22)}$ analiza la alegoría wachowskiana como reveladora de la dimensión ideológica que define cada vez más al capitalismo cognitivo contemporáneo, en particular, por mostrarse emblemática de las teorías conspirativas basadas en referencias seudocientíficas.

Atkin ${ }^{(23)}$ propone interpretar a la Conservaduría y sus laberintos como otra referencia ingeniosa a la famosa alegoría de la caverna de Platón, en la medida en que se trata de espacios paralelos y oscuros, unidos por la proyección de sombras que eventualmente se convierten en registros inertes de una realidad que sucede en planos exteriores iluminados. También el argumento de Matrix fue analizado como una cita evidente a la caverna de Platón ${ }^{(24,25)}$. En este sentido, Partridge $^{(25)}$ observa que las vidas dentro de la Matrix representan estados mentales completamente alienados de la realidad y que, irónicamente, la escenografía del mundo real es lo que más se asemeja en términos estéticos a la parte subterránea de una caverna.

En el libro VII de La República, Platón ${ }^{(26)}$ propuso una teoría general del conocimiento, basada en la distinción entre el mundo sensible y el mundo inteligible que se volvería uno de los fundamentos epistemológicos de la cultura occidental. A modo de ejemplo, el filósofo griego describió una caverna oscura poblada por humanos que ignoraban su condición de prisioneros desde el nacimiento. El único vínculo que tenían con el mundo exterior era la visión de sombras proyectadas en las paredes de la caverna. Platón postula que nuestro conocimiento sobre el mundo sensible siempre es incierto, independientemente de cuál sea la fuente de la información. Para él, lo que podemos aprehender no es lo propio del mundo sensible sino de un mundo inteligible del que surge nuestro mundo sensible conocido. Solo se puede conocer los fenómenos del mundo sensible luego de aprehender las formas del mundo inteligible. Para alcanzar la verdad de las cosas, entonces, no debemos dejarnos engañar por nuestros sentidos. Dado que el mundo común (real) se muestra demasiado nebuloso y cambiante como para permitir un conocimiento genuino, la conciencia de esta nebulosidad y de esta mutabilidad nos ayudarían a determinar qué percepciones y convicciones son relativamente más confiables.

Si bien hay convergencias y similitudes con la alegoría platónica, en las películas de la trilogía Matrix hay una mayor discontinuidad entre el mundo real y el simulado que la que existe entre la caverna y el mundo exterior de Platón. Partridge ${ }^{(25)}$ también opina que, a pesar de las diferencias metafísicas entre el pensamiento platónico original y el argumento de la trilogía Matrix, ambos convergen en lo que respecta a la inseguridad epistemológica del conocimiento objetivo y a la necesidad de superación de las limitaciones de los sentidos con el objetivo de alcanzar el conocimiento genuino. Este comentario también se puede aplicar al universo ficcional contenido en la figura de la Conservaduría ${ }^{(23)}$. Lo más intrigante es que, después de Todos los nombres, Saramago escribió un libro titulado La caverna ${ }^{(27)}$ en el que aborda la soledad de seres humanos presos de su ignorancia y alienados del mundo real y del control de sus propias vidas.

Sin embargo, no creo que la saga de Morfeo, Trinity y Neo o la obsesión de Don José sean un tema de interés inmediato para epidemiólogos o investigadores biomédicos, entre otros. Para la teoría epidemiológica, lo que importa es destacar que tanto la Matrix como la Conservaduría comprenden modelos 
reducidos de todo un universo y de toda una municipalidad que, al mismo tiempo, codifican y encapsulan poblaciones enteras. Todos los habitantes de estos mundos distópicos existen paralelamente en las estanterías y en las memorias de las ciclópeas bases de datos y cuentan con conexiones y redes (entre cuerpos y programas en el caso de la Matrix, y entre expedientes y fichas de vivos y de muertos, sepultados en archivos enmohecidos, en el caso de la Conservaduría).

Uno de los movimientos cruciales para la constitución histórica de la epidemiología, cuando era una joven disciplina en busca de un objeto, fue la organización de modelos reducidos de la dinámica real de los fenómenos de salud-enfermedad de la sociedad. En la década de 1950, la primera generación de líderes científicos de nuestro campo promovió el establecimiento de observatorios para la exploración metodológica, seleccionando poblaciones enteras para su monitoreo continuo y sistemático ${ }^{(28)}$. La ciudad de Framingham, en Nueva Inglaterra, es famosa aun hoy por haber sido el escenario de los principales hallazgos epidemiológicos con relación a los factores de riesgo cardiovascular, descubiertos por grupos de investigación vinculados a la Universidad de Harvard. Otras escuelas de salud pública implementaron sitios de observación similares, como Alameda County (en la Universidad de California en Los Ángeles), Evans County (en la Universidad de Carolina del Norte) y Hagerstown, Maryland (en la Universidad Johns Hopkins), entre otros ejemplos. Las poblaciones de esas localidades fueron monitoreadas por años (en algunos casos hasta el presente) examinando detalladamente sus condiciones de salud mediante exámenes periódicos clínicos y de laboratorio. Las muestras de fluidos y de tejidos producidas durante este proceso fueron almacenadas para futuras investigaciones. Esto implicó un enorme esfuerzo intelectual e institucional con el objetivo de modelar bases de datos y unidades de conservación como análogos físicos de las poblaciones humanas.

Tal como mencioné en otro texto ${ }^{(7)}$, estos muestrarios o modelos reducidos de comunidades o sociedades (compuestos con muestras demográficas, biológicas y clínicas, fragmentos de poblaciones reales recolectados, almacenados y expuestos) todavía se encuentran en funcionamiento y se los puede visitar en varios centros de investigación epidemiológica del mundo. Estos observatorios, que de una manera intrigante se asemejan a la alegoría de la Conservaduría de Saramago en términos de forma y función, contribuyeron mucho al desarrollo de los diseños de investigación y las técnicas de análisis que eventualmente se convertirían en el paradigma de la metodología científica observacional característica de la epidemiología ${ }^{(28)}$. Sin embargo, los límites de la composición y el tamaño físico, así como las limitaciones psicológicas, sociales, éticas, políticas e institucionales impuestas por la realidad de la vida cotidiana de las poblaciones concretas, impidieron que estas experiencias se transformaran en el "laboratorio epidemiológico natural" soñado por los fundadores de nuestra ciencia, especialmente Greenwood ${ }^{(29)}$.

El "sueño epidemiológico" se volvería realidad recién en la década de 1960 gracias a la introducción de la computación, cuya lógica de programación hizo posible el procesamiento electrónico de datos. Esto posibilitó que, como solución para modelar realidades epidemiológicas, se aplicaran simulaciones digitales, análogos estadísticos en situaciones concretas de estudios ambientales y poblacionales. Por primera vez fue posible organizar conjuntos de datos lo suficientemente grandes como para reproducir estructuras ecológicas y demográficas con dinámicas un poco más aproximadas a las de las poblaciones reales. Además, el control computarizado de datos aumentó el potencial de producción de conocimiento científico por la combinación de estrategias de observación con control analítico posterior. Cuando se las aplica a ciertos temas de investigación (como la salud en poblaciones humanas, una referencia crucial del objeto de la epidemiología), tales estrategias propician la transformación de individuos y grupos humanos (así como la de su estructura y dinámica biológica, demográfica y social) en registros y datos, mediante procesos de selección, codificación, traducción y compresión de significados. 
Teóricamente, estos procesos resultan en modelos flexibles o dispositivos artificiales que permiten simulaciones, fragmentaciones y reconstrucciones que, en la práctica, comprenden el verdadero sentido del análisis en este selecto campo de investigación científica. En términos objetivos y rigurosos, las únicas poblaciones concretas con las que tratan los científicos de este campo de investigación durante sus prácticas son las poblaciones de las bases de datos. Los investigadores epidemiológicos, en realidad, no se relacionan directamente con moléculas, células, tejidos, órganos o cuerpos humanos, sino que codifican, organizan, analizan y ponen en cuestión conjuntos artificiales de observaciones y datos, normalmente almacenados en formularios electrónicos. Desde una perspectiva metodológica rigurosa, la población objetivo de una investigación epidemiológica (construida por la práctica diaria de investigación, mediante la recopilación de observaciones, la codificación de datos y la producción de información) constituye un objeto abstracto, de naturaleza digital. En este sentido, se trata, grosso modo, de un entorno programable, interactivo y virtual de la misma naturaleza que la Matrix, aunque mucho menos complejo (de hecho, inmensamente más simple).

La población de los estudios epidemiológicos comprende una representación normalmente distante de la llamada población de referencia (y bastante diferente en términos de contenido y sustancia). En otras palabras, la población virtual de la epidemiología (o muestra) constituye un simulacro digital de una población de referencia compuesta por personas de carne y hueso. El término "simulacro" se utiliza en este caso en un sentido en parte adaptado, ya que no indica la mera imitación o copia vacía, sino más bien un símil de cada caso individual que conserva solamente algunos atributos dimensionales seleccionados a partir de su inmensa diversidad y complejidad biológica, ecológica, psicológica y cultural (es decir, un caso reducido a su medición).

El proceso de observación y producción de datos en trabajos de campo o de laboratorio constituye la única garantía confiable de la interrelación entre la muestra o la población de estudio y la realidad concreta. Esto significa que podemos confiar en que una base de datos analizada tiene como referencia o se corresponde con la población de un determinado distrito o ciudad solo si creemos que la metodología utilizada es rigurosa y adecuada. Esta garantía surge de un proceso de negociación sutil y complejo, casi un término tácito del acuerdo suscrito por los investigadores, miembros de redes más amplias de apoyo discursivo e institucional que funcionan como garantes de todo el proceso ${ }^{(30,31)}$.

Sin embargo, en estudios poblacionales de gran tamaño, el equipo que ejecuta las tareas de campo no siempre es el mismo que realiza el análisis de los datos, ni éste forma parte, muchas veces, del grupo que finalmente dará sentido a la información producida. Existen muchos ejemplos recientes de investigaciones en las que se subcontrata a empresas privadas para la recolección de los datos, por lo que la única cosa material que reciben y procesan los investigadores es un conjunto de datos que ya se encuentran en formato digital. Además, los epidemiólogos, como investigadores, no se relacionan directamente con el sufrimiento, la angustia, la enfermedad, la patología social, la migración, la vida, el nacimiento y la muerte, sino que se limitan a trabajar con datos, información y conocimiento que normalmente se traducen en criterios, parámetros, estimaciones y medidas de riesgo y sus correlaciones.

El concepto de riesgo y sus correlaciones se pueden interpretar como el elemento central del campo científico de la epidemiología(7). Más allá de las fronteras de esta disciplina científica, el concepto de riesgo se está convirtiendo cada vez más en una parte esencial del lenguaje de las ciencias biomédicas $y$ de la salud ${ }^{(3,32,33,34)}$. Esto implica admitir que cada uno de nosotros es portador de un perfil epidemiológico que puede representarnos en el mundo paralelo creado por el discurso epidemiológico. Este perfil implica, básicamente, configuraciones de factores de riesgo o, si usamos la reducción clínica del concepto original, configuraciones individuales de riesgo. La variante de la matrix creada por el discurso y la práctica de esta modalidad de 
ciencia puede representar, por lo tanto, puros "perfiles epidemiológicos", perfiles de sí y de todos, sujetos no-subjetivos, transhumanos.

Sin embargo, cabe señalar que no se trata de un proceso discursivo exclusivo de la ciencia epidemiológica. Otros discursos científicos, como el ecológico, el económico o el sociológico, también desarrollan teorías propias basados en las nociones de "individuo" y de "riesgo". En el caso de la epidemiología, la diferencia está en que esas nociones convertidas en conceptos permitieron construir una dimensión paralela de poblaciones formadas por seres ficticios contingentes, expuestos a riesgos, sujetos a la vida y vulnerables al sufrimiento, a las enfermedades y a la muerte. Con la ayuda del desarrollo de la estadística contemporánea, se puede reconocer, en principio, las matrices epidemiológicas de personas y eventos como poblaciones virtuales y estocásticas en la medida en que la posibilidad de que sus miembros se comporten o evolucionen de un modo diferente y singular está definida por probabilidades individuales de enfermar o de morir.

\section{EL HOMBRE DE LOS RIESGOS RELOADED}

A estas alturas quisiera ratificar la proposición de que el concepto epidemiológico de riesgo implica un entorno virtual habitado por poblaciones abstractas, regidas por parámetros y normas funcionales que son manipulados analíticamente siguiendo protocolos particulares y algoritmos preestablecidos. Quizás ahora, en la era de la realidad aumentada, las monedas digitales, las redes sociales y la inteligencia artificial, podamos vislumbrar con mayor claridad esta dimensión especial (casi un universo paralelo) formada por sustitutos de poblaciones humanas compuestos de simulacros transhumanos de sujetos humanos. Una lógica inferencial probabilística, bastante desarrollada y actualmente cultivada por diferentes ciencias, regula la conexión y la articulación que existen entre los dos niveles (el real y el virtual, el analógico y el digital) de la indagación tecnocientífica responsable de la efectividad técnica y práctica de la disciplina epidemiológica ${ }^{(34)}$.

El "sueño epidemiológico", sobre terreno fértil, tendría al fin su contraparte justa en el imaginario social contemporáneo, alimentado por una sociedad que se presenta como posmoderna, compuesta por redes, conexiones, híbridos, clones y duplicados. No sorprende, entonces, que un entorno metafórico como el descripto, con su peculiar ecología social de los riesgos, se vuelva cada vez más real, en el sentido de que se le ha concedido una existencia material. Esto es así porque, con el big data, se está construyendo realmente una dimensión paralela que tiene sus propias condiciones de producción y reproducción de efectos en la vida social e histórica concreta.

Hace casi tres décadas, cuando escribí el ensayo "El hombre de los riesgos" que cité en la introducción ${ }^{(6)}$, utilicé como ilustración general de esa realidad el uso de la tarjeta de crédito, una opción bancaria popular que era nueva en ese entonces. Afirmé que, al Ilenar un formulario de inscripción para disponer del dinero, las personas pasarían a tener una existencia paralela en una base de datos que representaría una reducción del conjunto de las relaciones económicas, y que esta existencia estaría regida solo por la racionalidad económica más simple del balance de débitos y créditos. Consumir sin utilizar dinero en efectivo es cada vez más fácil. Cualquier persona puede adquirir ropa o medicamentos, hacer compras, contratar servicios o personalizar productos, y pagar con una tarjeta magnética o virtual, deduciendo los gastos directamente de su capacidad económica, saldo bancario o potencial crediticio. Sin embargo, para el registro y el procesamiento de operaciones bancarias o financieras, el crédito o el poder adquisitivo no pertenece directamente a la persona, sino que refiere al otro del sujeto humano, al doble transhumano o al identificador numérico activado por una clave (en general alfanumérica) que habita los bancos de memoria y circula por los circuitos de las redes de información financiera.

En los países con una economía industrial avanzada, en los que impera el ya citado 
capitalismo cognitivo ${ }^{(10)}$, los sujetos están acostumbrados desde hace mucho tiempo a esa existencia paralela, que opera en un mundo ficticio de bases de datos cada vez más interconectadas. Comenté anteriormente que, en el ámbito profesional, los solicitantes de empleo se encuentran registrados en redes de información del mercado laboral organizadas para generar datos sobre competencias, antecedentes laborales y referencias profesionales, las cuales están interconectadas con los sectores de recursos humanos de megaempresas. También mencioné que, en el campo de la salud, los valores de las primas y de las pólizas de los seguros de salud, así como las condiciones de tratamiento a las que tienen derecho los sujetos que los contratan, se definen de acuerdo a algoritmos que calculan potenciales de salud individual a partir de probabilidades de enfermar, o sea, a partir de los perfiles de riesgo de los sujetos ${ }^{(35)}$.

En "El hombre de los riesgos"(6), destaqué otra convergencia teórica sobre quién sería ese transhumano, uno y múltiple. Se trata de la cuestión de la supuesta identidad del sujeto, que emerge como efecto de ese discurso, y que acaba revelando, por el lado de la patología, un ser que no es más el sujeto que conocemos. Hice referencia al clásico Las palabras y las cosas, en el que Foucault ${ }^{(36)}$ propone que el hombre moderno (figura mítica que, por su singularidad, volvió posible la referencia universalizante del discurso científico) había sido inventado en el siglo XVIII. Allí comenté que el nuevo sujeto de la posmodernidad estaría siendo inventado tal vez en ese momento, como un "hombre unidimensional" determinado por probabilidades de eventos ${ }^{(37)}$. Este nuevo ser ya no sería un sujeto, o un hombre moderno en el sentido foucaultiano, por portar una parte nosubjetiva (su doble o doppelgänger), la cual habita en redes de información, tal como lo demuestran sus números de registro computarizados (de índole civil, electoral, crediticio, de seguridad social, etc.) y sus claves. Desde una perspectiva ampliada, este nuevo ser, mitad humano y mitad máquina, tanto material como digital, sería un cyborg, según la propuesta de Donna Haraway, musa intelectual del posmodernismo californiano. En sus palabras $^{(5)}$, ese transhumano sería:

...una criatura en el mundo posgénero [...] comprometida decididamente con la parcialidad, la ironía, la intimidad y la perversidad; [...] sin nada de inocencia. La polaridad entre lo público y lo privado ya no estructura al cyborg, que define una polis tecnológica basada de forma parcial en la revolución de las relaciones personales.

El cerebro cyborg opera como una red o, cuando está conectado, como un avatar o un personaje en la Matrix, y su subjetividad asume una forma diferente en dos sentidos. En primer lugar, al estar los seres humanos interconectados por el idioma y la cultura, existe un elemento peculiar, definido simbólicamente, que depende de los lenguajes y los programas compartidos. $Y$, en segundo lugar, es básicamente necesario, sobre todo para las máquinas humanas, tener un socius que esté establecido relacionalmente y conectado en red. A través de ambos elementos -la relevancia cultural y el vínculo social común- se realiza la descarga (download) de la "realidad" en cada cerebro. Morfeo describe esto como "having a dream", que sería el estado de todos los seres dentro de la Matrix. Por su parte, Don José también se pregunta cómo se puede distinguir entre el mundo de los sueños y el mundo real de la Conservaduría. Esta línea de cuestionamiento aparece en muchas discusiones filosóficas y obras literarias que buscan generar una distinción entre los estados de sueño y de realidad, lo que conduce inmediatamente a problemas en la "presentación del mundo", para usar una expresión acuñada por Giannotti( ${ }^{(38)}$.

Hoy en día, la conexión necesaria entre las distintas partes y las diferentes existencias de este nuevo ser está determinada por operaciones diversas e insignificantes de la vida cotidiana, como una simple compra por Internet, una búsqueda en un navegador, el uso de terminales bancarios o comerciales automatizados o, de una manera aún más actual y ciertamente más común, mediante una 
aplicación en un dispositivo de conectividad denominado "inteligente": el smartphone. Estos ejemplos ilustran la informatización progresiva de nuestra vida cotidiana, durante la cual las redes de medios de comunicación electrónicos capturan cada vez más a los sujetos. Žižek ${ }^{(17)}$ considera que, en este proceso, los individuos están expuestos a la amenaza de una alienación radical: los sujetos terminan potencialmente reducidos a un vacío puro, ya que el conjunto de sus recuerdos y su propia experiencia personal pueden ser regulados, manipulados, o incluso borrados o robados, por lo que él llama el "otro maquínico", que bien podría ser otro nombre para la Matrix. En el último tiempo, Žižek ${ }^{(39)}$ retomó este enfoque interpretativo al proponer que la Matrix representa nada menos que el gran otro de la teoría psicoanalítica lacaniana, indicativo de la alienación constitutiva del sujeto en el orden simbólico.

El tema de la salud-enfermedad se vuelve fundamental en este momento histórico de constitución de una nueva figura mítica (el sujeto de los riesgos, el transhumano de la posciencia) tal como lo fue en el contexto de la invención del hombre que se produjo con El nacimiento de la clínica durante el surgimiento de la ciencia moderna. En este momento verificamos que el concepto de Riesgo (con R mayúscula, en el sentido estructurante de la vida transhumana), con toda su complejidad simbólica y riqueza analítica ${ }^{(33)}$, acaba revelando, por el lado de la patología, un ser humano que ya no es el que conocemos desde hace siglos, sino que es el primero de una nueva generación de sujetos posclínicos.

En resumen, este sujeto, transhumano, "criatura en el mundo posgenérico"(5), parcialmente digital, computarizado y mensurado, de gustos escrutados y deseos prospectados, está cada vez más marcado, definido y condicionado por la probabilidad de ocurrencia de todo tipo de evento de la vida, incluso los de salud. Con relación a este tipo de eventos, lo que se comparte con otros miembros de una población, en términos epidemiológicos, es el Riesgo, un concepto que parece haber sido acuñado, desarrollado y personalizado para hablar sobre la patología colectiva transhumana. Por todo esto, el Riesgo es un elemento fundante de la construcción del cyborg como figura mítica históricamente constituida y conceptualmente representativa de los tiempos actuales.

\section{EPÍLOGO: LA MATRIX DE TODOS LOS RIESGOS}

En realidades totalizantes como la Conservaduría, la Matrix y el "sueño epidemiológico" se verifica una reducción de los sistemas, fuerzas, agentes y actores que componen estructuras complejas a elementos simples y conexiones lineares, bajo niveles de organización más rígidos. Restablecer a través de teorías, análisis y modelados la naturaleza caótica y la complejidad del mundo concreto, biológico, social e histórico, constituye una tarea importante para quienes construyen la ciencia en lo cotidiano de sus investigaciones. De hecho, estos dispositivos teóricos (o modelos) son imprescindibles para abordar metodológicamente la complejidad opresiva de las "realidades reales" de las disciplinas científicas ${ }^{(40)}$.

Utilizamos, específicamente, metáforas (dispositivos imaginarios, realidades abstractas o entornos teóricos) que por definición posibilitan el proceso de investigación científica. Esta operación metafórica no es exclusiva de la ciencia epidemiológica, sino que se pueden aplicar analogías equivalentes a los mundos demográficos, económicos, ecológicos y meteorológicos como efecto de las respectivas disciplinas científicas de la Demografía, Economía, Ecología y Meteorología. ¿Acaso alguien podría defender que "una generación", "el medio ambiente", "el mercado" o "el clima" son objetos tangibles o materiales, y no entidades metafóricas, abstractas o virtuales?

Al leer Todos los nombres de Saramago y al ver Matrix de las hermanas Wachowski, se puede vislumbrar un paisaje virtual epidemiológico que es más real de lo que parece y más imaginario de lo que se podría imaginar. Esta percepción se basa en una posibilidad primaria, la cual se propone como hipótesis: 
la Conservaduría y la Matrix constituyen alegorías que pueden interpretarse como equivalentes a los mundos virtuales construidos por las vertientes moderna y posmoderna de la epidemiología, respectivamente. Ambas alegorías son ilustrativas del mundo virtual posibilitado por la tecnociencia, en particular en los procesos de producción de conocimiento por medio de estrategias observacionales y meta-analíticas, como es el caso de la ciencia epidemiológica.

En el presente ensayo examiné la consistencia y la validez potencial de esta hipótesis recurriendo a los primeros observatorios sociales que usaron poblaciones enteras para su observación sistemática, lo que permitió el surgimiento del "sueño epidemiológico", ahora potenciado por el avance de estrategias de modelado y simulación, y por la organización de megabases de datos sobre salud, enfermedad, vida y muerte. Propuse una articulación entre el concepto epidemiológico de riesgo y una concepción de ambiente (metafórico, virtual, habitado por poblaciones abstractas) que es propia de la epidemiología. Argumenté que los epidemiólogos tratan en su práctica concreta con la población imaginaria de sus bases de datos, a las que solo el proceso de producción de datos les puede conceder alguna garantía de referencia mediante un proceso de negociación sutil y complejo que constituye un compromiso implícito firmado por los investigadores implicados. Podemos concluir que, de hecho, la población real de referencia es distinta a la virtual y abstracta construida en la práctica cotidiana de investigación. La conexión entre esos dos niveles está regida por la lógica inferencial particular desarrollada por la epidemiología, la cual es responsable de la eficacia técnica de esta disciplina. A partir de esto surgió una práctica social denominada medicina preventiva, con el objetivo de anticipar la ocurrencia de eventos indeseables (básicamente enfermedad y muerte) en poblaciones abstractas indicadas como portadoras de riesgos. Con este fin, se invirtió una cantidad enorme de tiempo y energía en la invención de nuevas lógicas (y nuevas éticas) para la gestión de realidades paralelas construidas a partir de la noción de riesgo ${ }^{(41)}$.
Cuando concebí la alegoría del hombre de los riesgos, propuse distinguir diferentes modalidades del concepto de riesgo: el riesgo poblacional (concepto epidemiológico en sentido estricto), el peligro o amenaza (noción latente u oculta en el discurso social común) y el riesgo individual (concepto práctico incorporado por la clínica) ${ }^{(6)}$. Hace exactamente 10 años, apreciamos que el futuro del concepto de riesgo dependería de su capacidad de acompañar los desarrollos conceptuales y metodológicos de las nuevas ciencias de la salud, contribuyendo con modelos teóricos y estrategias empíricas capaces de abordar los complejos objetos emergentes $^{(3)}$. En este sentido, propuse incorporar dos definiciones más a la lista de conceptos de riesgo: el riesgo ambiental (probabilístico, aunque no individual) y el riesgo estructural (posibilístico). Poco después, al sistematizar nuevas categorías de determinación con el fin de renovar la discusión epistemológica sobre la epidemiología, introdujimos la idea de riesgo contingencial al glosario de riesgos para incorporar eventos singulares, accidentales o catastróficos ${ }^{(34)}$.

Reitero lo que ya propuse anteriormente: el "sujeto de los riesgos" será, ciertamente, menos humano. Tal vez sea más correcto denominarlo transhumano. Técnicamente, se volverá cada vez más difícil reconocer qué tendrá el transhumano -ese cyborg inmaterial- de subjetivo, singular, personal, humano, identitario o particular y será más fácil definirlo por lo que comparta con todos los otros. Y lo que se puede compartir en este nuevo mundo probabilístico y totalizante son repertorios de posibilidades de acontecimientos y sucesos, registrados desde una perspectiva frecuentista, y reconocidos y predichos en algoritmos en los lenguajes de programación.

Por último, debo destacar dos acontecimientos que han producido un cambio de escenario sustancial (aunque previsto) desde "El hombre de los riesgos". Por un lado, el gran aumento en la capacidad compartida de procesamiento y almacenamiento entre computadoras y sus redes de sistemas distribuidos permitió el advenimiento de lo que se 
ha denominado Big Data. Por otro lado, se verificó un rápido avance en la llamada inteligencia artificial, que consiste en la capacidad de autoajuste constante en la programación de dispositivos electrónicos para el tratamiento de la información (lo que, con un grado reducido de rigor semántico, se denominó aprendizaje automático). En realidad, se trata de algoritmos que componen el lenguaje de máquina, y que son capaces de reprogramarse a sí mismos o de autorregularse por medio de una operación de análisis de megabases de datos a una velocidad de procesamiento altísima (que hoy en día tiende a ser incluso mayor, con la prometida computación cuántica). El aumento en la velocidad de procesamiento y la mayor capacidad para almacenar datos y automatizar estrategias de análisis permiten integrar las diferentes formas de riesgo, lo que de cierto modo resucita al "demonio de Laplace", amo de un mundo totalmente previsible. Solo las singularidades y las contingencias inevitables desafiarán a este universo programado, embrión de una Matrix menos increíble, aunque no por eso menos peligrosa.

En la actualidad, se observa en todo el mundo un interés creciente por las técnicas y los procedimientos destinados a mejorar a los sujetos humanos, transformándolos en organismos (o ciberorganismos), perfiles de riesgo puros, determinados por funciones, factores, marcadores, comportamientos y situaciones de riesgo, atribuidos a sujetos no-subjetivos con existencia en mundos virtuales conformados como matrices de riesgo. Esto implica admitir que se puede plantear una imagen posible de la epidemiología como un discurso científico mucho más poderoso de lo que la mayoría de nuestros tímidos epidemiólogos se acostumbró a pensar, aun sin necesidad de tomar la píldora roja y seguir al conejo blanco (como hizo Neo) o de copiar la ficha de la mujer desconocida (como hizo Don José). Muchas personas siguen intrigadas y deslumbradas al comprobar que la epidemiología, como ciencia y como práctica, está de moda.

La evaluación de la hipótesis central de este ensayo refuerza la tesis de que, ante este sujeto transhumano, posclínico, posgénero y cyborg, la ciencia epidemiológica es capaz de asumir un papel central equivalente al que desempeñaron las ciencias biomédicas con relación al sujeto de la modernidad. Como bien nos enseñó Foucault ${ }^{(42)}$, la medicina clínica fue el primer discurso técnico sobre el hombre en la modernidad, que tiende a desvanecerse con la superación de este sujeto obsoleto. De esta manera, puedo actualizar aquí la propuesta de que la epidemiología definida como un discurso privilegiado sobre los riesgos individuales y colectivos, productora de aportes teóricos y técnicos capaces de ampliar y profundizar la comprensión de los riesgos ambientales, estructurales y contingentes- cumple con todos los requisitos necesarios para convertirse en la ciencia fundamental de la salud en la era posclínica.

En resumen, hay algo en nuestra ciencia que la hace un elemento importante en la cosmología de los tiempos que una vez se Ilamaron postmodernidad. ¿Qué cosa es?, "ya tienes la respuesta", diría Morfeo. Y añadiría: "no sabes lo que es, pero está ahí, como una espina en tu mente". Esta vaga percepción de incomodidad (que no implica más que un sentimiento) nos hace pensar que esto es así porque los discursos de la joven ciencia epidemiológica tienen un poder enorme para sintonizar con los tiempos históricos. Esto es lo que permite entender por qué la epidemiología nació fuera de la caverna, salió hace mucho tiempo de la Conservaduría, y se encuentra ahora en la Matrix. Ahora es cuestión de volver posible lo necesario: una conciencia histórica de las redes de imágenes, sentidos y saberes que estructuran y limitan, dentro y fuera de la matriz, a una nueva ciencia de los riesgos en un mundo transhumano. 


\section{AGRADECIMIENTOS}

Este texto corresponde a una contribución para el seminario "As várias faces do risco e os desafios da educação na sociedade contemporânea" ("Las distintas caras del riesgo y los desafíos de la educación en la sociedad contemporánea"), dictado en el Instituto de Estudios Avanzados de la Universidad de São Paulo, el 15 de octubre de 2019, cuya versión final recibió la valiosa revisión crítica de Diego Almeida y Denise Coutinho.

\section{REFERENCIAS}

1. Diaz E. La posciencia: el conocimiento científico en las postrimerías de la modernidad. Buenos Aires: Biblos; 2007.

2. Castoriadis C. Science moderne et interrogation philosophique. En: Les Carrefours du Labyrinthe. Paris: Seuil; 1978.

3. Almeida Filho N, Castiel L, Ayres J. Riesgo: concepto básico de la epidemiología. Salud Colectiva. 2009;5(3):323-344. doi: 10.18294/sc.2009.238.

4. Gadamer G. The enigma of health. Stanford: Stanford University Press; 1996.

5. Haraway D. Symians, cyborgs, and women: The reinvention of nature. New York: Routledge; 1991.

6. Almeida-Filho N. A clínica e a epidemiologia. Rio de Janeiro: APCE, Abrasco; 1992.

7. Almeida-Filho N. La ciencia tímida: Ensayos hacia la deconstrucción de la epidemiología. Buenos Aires: Lugar Editorial; 2000.

8. Sevalho G. O "homem dos riscos" e o "homem lento" e a teorização sobre o risco epidemiológico em tempos de globalização. Interface - Comunicação, Saúde, Educação. 2012;16(40):7-19. doi: 10.1590/S1414-32832012005000005.

9. Portella SL, Oliveira SS, Valencio N, Nunes JA. Da "ponte sobre águas turbulentas" à reinvenção do "homem lento": reflexões sobre assimetrias de saber e desastres. Revista Ciência \& Trópico. 2016;37(1):1-22.

10. Boutang YM. Cognitive capitalism. London: Polity Press; 2011.

11. Chalmers A. What is this thing called Science? Londres: Open University Press; 1982.

12. Bhaskar R. Scientific realism and human emancipation. London: Verso; 1986.
13. Saramago J. Todos os nomes. São Paulo: Companhia das Letras; 1997.

14. Almeida-Filho N. Saramago's All the Names and the epidemiological dream. Journal of Epidemiology and Community Health. 2004;58(9):743746.

15. Houaiss A. Dicionário Houaiss da Língua Portuguesa. Rio de Janeiro: Editora Oliveira; 2001.

16. Rey A. Dictionnaire Historique de la Langue Française. Paris: Dictionnaires Le Robert; 1993.

17. Horsley J. Matrix warrior: being the one. New York: Thomas Dunne Books; 2015.

18. Žižek S. The Matrix: The two sides of perversion [Internet]. 1999 [citado 15 oct 2019]. Disponible en: https://tinyurl.com/tb68zxe.

19. Manzotti R. The computational stance is unfit for consciousness. International Journal of Machine Consciousness. 2012;4(2):401-420.

20. Hanley R. Reflexions on the First Matrix. En: Philosophy \& The Matrix [Internet]. 2002 [citado 15 oct 2019]. Disponible en: https://tinyurl.com/ uux4mam.

21. Bostrom N. Are you living in a computer simulation? Philosophical Quarterly. 2003;52(211):243255.

22. Paura R. Living in the Matrix: How a scientific conjecture was turned into a conspiracy theory. Messages, Sages and Ages. 2017;4(2). doi: 10.1515/msas-2017-0006.

23. Atkin R. From Theseus to Daedalus: Saramago, Sr. José, and the reader in the labyrinth of Todos os Nomes. Portuguese Studies. 2007;23(2):191-207.

24. Irwin W, (ed.). The Matrix and philosophy: welcome to the desert of the real. New York: Open Court Publishing Company; 2002.

25. Partridge J. Plato's Cave and the Matrix. En: Philosophy \& The Matrix [Internet]. 2003 [citado 15 oct 2019]. Disponible en: https://tinyurl.com/ rz97suv.

26. Platón. La República. Madrid: Akal; 2009.

27. Saramago J. A caverna. São Paulo: Companhia das Letras; 2000.

28. Susser M. Epidemiology in the United States after World War II: The evolution of technique. Epidemiologic Reviews. 1985;7:147 177. 
29. Greenwood M. Epidemiology: historical and experimental. London: Humphrey Millford; 1932.

30. Santos BS. Introdução a uma Ciência Pós-Moderna. Rio de Janeiro: Graal; 1989.

31. Latour B. Pandoras's hope: Essays on the reality of sciences studies. Cambridge: Massachusetts, Harvard University Press; 1999.

32. Vineis P. Modelli di rischio: epidemiologia e causalitá. Torino: Einaudi; 1990.

33. Hayes M. On the epistemology of risk: language, logic and social science. Social Science and Medicine. 1992; 35(4):401-407.

34. Coutinho D, Almeida-Filho N, Castiel LD. Epistemologia da epidemiologia (categorias de determinação: causalidade, predição, contingência, sobredeterminação). En: Almeida-Filho N, Barreto M, (org.). Epidemiologia \& saúde: fundamentos, métodos, aplicações. Río de Janeiro: Guanabara Koogan; 2011. p. 29-42.

35. Alexander J. The ideological construction of risk: an analysis of corporate health programs in the 1980s. Social Science and Medicine. 1988;26(5): 559-567.

36. Foucault M. Les mots et les choses: une archéologie des sciences humaines. París: Gallimard; 1966.

37. Marcuse H. One dimensional man. New York: Routledge; 1968.

38. Giannotti JA. Apresentação do Mundo. São Paulo: Companhia das Letras; 1995.

39. Žižek S. Cogito in the History of Madness [Internet]. 2019 [citado 15 oct 2019]. Disponible en: https://tinyurl.com/r8quxo3.

40. Bunge M. Method, model and matter. Dordrecht: Reidel Publishing Company; 1973.

41. Castel R. La gestion des risques. Paris: Editions de Minuit; 1981.

42. Foucault M. La naissance de la clinique. Paris: Gallimard; 1963. 